\title{
Interactive comment on "Spaceborne potential for examining taiga-tundra ecotone form and vulnerability" by P. M. Montesano
}

\section{P. M. Montesano}

paul.m.montesano@nasa.gov

Received and published: 11 April 2016

\section{Responses to RC2}

Comment 1 . The title of this manuscript is to examine the ecotone form and vulnerability. But the author did not specify or provide definitions in the paper what the form and vulnerability are (vulnerability was mentioned until the end of the manuscript). The form and vulnerability needs to be clearly specified in this study. For example, Page 3 line 20, "recent work notes that rapid growth changes forms. . ." It is vague what the form here means. Does it refer to individual stand or patch scale increase in height? In some other places, it reads as the form of patch size and distribution. Additionally, the authors need to specify what factors the TTE may be vulnerable to.

Response: The Reviewer suggests a the need to more clearly define form and vulner- 
ability up front, and points out that a vague reference to form appears (pg 3, line 20) before it is defined (pg 4, line 16). We will fix these incoherences in the next version.

Comment 2. Page 3 line 26-27, depending how extensive Taiga vegetation distributed, the height and relation with permafrost temperature actually varies (Roy-Levillee et al 2014). Double-check with the reference please

Response: The Reviewer is correct and we will edit the manner in which we reference that study to more accurately reflect that the variation in permafrost temperature is controlled in part by vegetation height, but also by the arrangement of taiga patches.

Comment 3. Page 8 line 11, first time DSM is mentioned here, please spell out.

Response: Page 8 line 11: We will insert 'digital surface model' here before 'DSM'

Comment 4. It seems that NDVI was used as a mask to determine whether the land cover is vegetated or not. It is not clear how the threshold was selected though. It will also be good to discuss/introduce roughness based on panchromatic HRSI image. Also discuss why this method can be useful without modification based on Johansen et al 2014.

Response: RC2 requests more information on the NDVI threshold used to separate vegetation from non-vegetation. Please see Comment \#2/Response to Reviewer \#1 and Comment \#6/Response to Reviewer \#4.

Comment 5. For study region, the authors mentioned that the study area was exclusively covered by one single boreal species Larix gmelini. Please clarify if this is also the case for the verification and validation sites. It will be good to note what the tall shrub species/tundra plant communities are. This study looks at forest-tundra ecotone, but shrub species are just left out, which might also be tall and these may be the ones respond to warming and changes patch dynamics.

Response: Both the study region where forest patches were mapped and the verification and validation sites featured the same forest type; exclusively Larix gmelini. We

Printer-friendly version

Discussion paper 
think the Reviewer makes a good point in suggesting we include some information on tall shrub species and tundra communities. We will add this information to the Study Area section. We will also note that we do not directly address shrub structure, as our field data do not include shrub measurements. However, our remote sensing analysis may include tall shrubs that may persist within the forest mask, and thus, a component of the patch height and uncertainty estimates may include shrub information. This warrants mention in the Discussion.

Comment 6. The Patch-based analysis sounds very straight forward and will reveal the local scale dynamics in TTE patches. However, it will be good to include a clear definition of patch as well. Maybe based on remote sensing texture characteristics "patch" seems to make sense. But how does it correlate to ecological meaning?

Response: Here we use the term 'forest patch' to refer to a group of trees that are relatively homogenous in terms of height and consistent in terms of horizontal arrangement. We will include text in Section 1.2 to clearly state what we mean by 'forest patch': The spatial configuration of tree of similar structure can be conceptualized as 'forest patches', whereby a patch represents a group of trees that exhibit relatively homogenous or consistent vertical and horizontal structure.

Interactive comment on Biogeosciences Discuss., doi:10.5194/bg-2015-575, 2016. 